\title{
Common Fixed Point Theorems of Multivalued Operators in Generalized Metric Spaces
}

\author{
Prof. Hiremath Suresh Shivarudrappa ${ }^{1,}$ Dr. Kakde Rameshkumar \\ Vishwambharrao ${ }^{2}$ \\ Brahmdevdada Mane Institute of Technology, Solapur(Maharashtra), India \\ Shri Sant Gadage Maharaj Mahavidyalaya, Loha(Maharashtra), India
}

\begin{abstract}
The purpose of this article is to obtain common fixed point theorems of multivalued operators in generalized metric spaces.

Keywords: multivalued mapping, fixed point, common fixed point, generalized metric space.

AMS Subject Classification (2000): 47H04, 47H10, 54 H25.
\end{abstract}

\section{Introduction:}

The concept of D-metric spaces was initiated by B C Dhage. The study was further enhanced by B E Rhoades, B C Dhage, A M Pathan.

Definition1.1: A non-empty set $X$ together with a function $D: X \times X \times X \rightarrow\left[\begin{array}{ll}0 & \infty\end{array}\right)$ is called a D-metric space, denoted $\langle X D\rangle$ if D satisfies

i) $D(x, y, z)=0$ if and only if $x=y=z \quad$ (coincidence)

ii) $D(x, y, z)=D(p\{x, y, z\})$ where pis a permutation of $x, y, z \quad$ (symmetry) iii)

$$
D(x, y, z) \leq D(x, y, a)+D(x, a, z)+D(a, y, z) \text { for } x, y, z, a \in X \quad \text { (tetrahedral inequality) }
$$

The non-negative real function $\mathrm{D}$ is called a D-metric on $X$. A $\mathrm{D}$-metric is called generalized metric on $X$ and the pair $\langle X D\rangle$ is called Generalized metric space.

Generally the usual ordinary metric is called the distance function. D-metric is called diameter function of the points of $X$.

The common fixed point theorems for multivalued mappings in metric spaces have been obtained by Alina Sintamarian[1] which improve and generalize a result given by A. Latif and I. Beg in [2]. Here we make use of the following theorem to obtain common fixed point theorems of multivalued operators in generalized metric spaces.

Theorem 2.1 Let $\langle X D\rangle$ be a metric space and $S, T: X \rightarrow P(X)$ be two multivalued operators. We suppose that at least one of the following condition is satisfied:

(i) there exists $\varphi: R_{+} \rightarrow R_{+}$a function with the property that $\varphi(0)=0$ and such that for each $x \in X$, any $u_{x} \in S(x)$ and for all $y \in X$, there exists $u_{y} \in T(y)$ so that we have

$$
d\left(u_{x}, u_{y}\right) \leq \varphi(d(x, y))
$$

ii) there exists $a_{1}, a_{2} \ldots ., a_{5} \in R_{+}$, with $a_{3}+a_{4}<1$ such that for each

$x \in X$, any $u_{x} \in S(x)$ and for all $y \in X$, there exists $u_{y} \in T(y)$ so that we have

$d\left(u_{x}, u_{y}\right) \leq a_{1} d(x, y)+a_{2} d\left(x, u_{x}\right)+a_{3} d\left(y, u_{y}\right)+a_{4} d\left(x, u_{y}\right)+a_{5} d\left(y, u_{x}\right)$

iii) there exists $a \in R_{+}$, with $a<1$ such that for each

$x \in X$, any $u_{x} \in S(x)$ and for all $y \in X$, there exists $u_{y} \in T(y)$ so that we have

$d\left(u_{x}, u_{y}\right) \leq a \max \left\{d(x, y), d\left(x, u_{x}\right), d\left(y, u_{y}\right), 1 / 2\left[d\left(x, u_{y}\right)+d\left(y, u_{x}\right)\right]\right\}$ 
iv) there exists $\varphi: R_{+}^{5} \rightarrow R_{+}$a function with the property that $\varphi(0,0, t, t, 0)<t$, for all $t>0$ and such that for each $x \in X$, any $u_{x} \in S(x)$ and for all $y \in X$, there exists $u_{y} \in T(y)$ so that we have $d\left(u_{x}, u_{y}\right) \leq \varphi\left(d(x, y), d\left(x, u_{x}\right), d\left(y, u_{y}\right), d\left(x, u_{y}\right), d\left(y, u_{x}\right)\right)$

Then $F_{S} \subseteq F_{T}$.

Definition 1.2 Let $X$ be a non empty set. By $P(X)$ we shall understand the set of all non empty subsets of $X$ . A correspondence $T: X \rightarrow P(X)$ is called a multivalued mapping on $X$.

Definition 1.3 A fixed point of multivalued mapping $T: X \rightarrow P(X)$ is a point $x \in X$ such that $x \in T(x)$

We denote by $F_{T}$ the set of the fixed points of $T$.

Let $\left\{T_{n}\right\}_{n \in N}$ be a sequence of multivalued operators with nonempty values that is $T_{n}: X \rightarrow P(X)$ for $n \in N$.

We denote by $\operatorname{ComFP}(T)$ the set of the common fixed points of the multivalued operators $T_{n}$, for $n \in N$

That is $\operatorname{ComFP}(T)=\left\{x \in X \mid x \in T_{n}(x)\right.$, for all $\left.n \in N\right\}=\bigcap_{n \in N} F_{T_{n}}$

Main Result

Theorem 2.2 Let $\left\langle\begin{array}{ll}X & D\end{array}\right\rangle$ be a generalized metric space and $S, T: X \rightarrow P(X)$ be two multivalued mappings. We suppose that at least one of the following condition is satisfied.

(i) there exists $\varphi: R_{+} \rightarrow R_{+}$a function with the property that $\varphi(0)=0$ and such that for each $x \in X$, any $u_{x} \in S(x)$ and for all $y \in X$, there exists $u_{y} \in T(y)$ so that we have

$$
D\left(u_{x}, u_{y}, u_{y}\right) \leq \varphi(D(x, y, y))
$$

ii) there exists $a_{1}, a_{2} \ldots . ., a_{5} \in R_{+}$, with $a_{3}+a_{4}<1$ such that for each

$x \in X$, any $u_{x} \in S(x)$ and for all $y \in X$, there exists $u_{y} \in T(y)$ so that we have $D\left(u_{x}, u_{y} u_{y}\right) \leq a_{1} D(x, y, y)+a_{2} D\left(x, u_{x}, u_{x}\right)+a_{3} D\left(y, u_{y}, u_{y}\right)+a_{4} D\left(x, u_{y}, u_{y}\right)+a_{5} D\left(y, u_{x}, u_{x}\right)$

iii) there exists $a \in R_{+}$, with $a<1$ such that for each $x \in X$, any $u_{x} \in S(x)$ and for all $y \in X$, there exists $u_{y} \in T(y)$ so that we have $D\left(u_{x}, u_{y}, u_{y}\right) \leq a \max \left\{D(x, y, y), D\left(x, u_{x}, u_{x}\right), D\left(y, u_{y}, u_{y}\right), 1 / 2\left[D\left(x, u_{y}, u_{y}\right)+D\left(y, u_{x}, u_{x}\right)\right]\right\}$ iv) there exists $\varphi: R_{+}^{5} \rightarrow R_{+}$a function with the property that $\varphi(0,0, t, t, 0)<t$, for all $t>0$ and such that for each $x \in X$, any $u_{x} \in S(x)$ and for all $y \in X$, there exists $u_{y} \in T(y)$ so that we have $D\left(u_{x}, u_{y}, u_{y}\right) \leq \varphi\left(D(x, y, y), D\left(x, u_{x}, u_{x}\right), D\left(y, u_{y}, u_{y}\right), D\left(x, u_{y}, u_{y}\right), D\left(y, u_{x}, u_{x}\right)\right)$

Then $F_{S} \subseteq F_{T}$.

Proof: We assume that condition (i) is satisfied

Let $x^{*} \in F_{S}$ Then $x^{*} \in S\left(x^{*}\right)$ and it follows that there exists $u \in T\left(x^{*}\right)$ such that

$$
D\left(x^{*}, u, u\right) \leq \varphi\left(D\left(x^{*}, x^{*}, x^{*}\right)\right)=\varphi(0)=0
$$

This implies that $x^{*}=u$

Therefore, $x^{*} \in T\left(x^{*}\right)$ and hence $F_{S} \subseteq F_{T}$

Now suppose that the condition (ii) is satisfied.

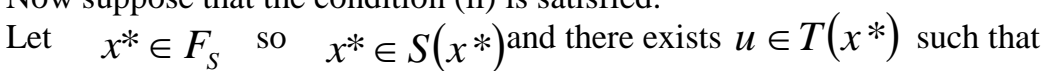




$$
\begin{aligned}
D\left(x^{*}, u, u\right) & \leq a_{1} D\left(x^{*}, x^{*}, x^{*}\right)+a_{2} D\left(x^{*}, x^{*}, x^{*}\right)+a_{3} D\left(x^{*}, u, u\right)+a_{4} D\left(x^{*}, u, u\right)+a_{5} D\left(x^{*}, x^{*}, x^{*}\right) \\
& =\left(a_{3}+a_{4}\right) D\left(x^{*}, u, u\right)
\end{aligned}
$$

This implies $x^{*}=u$

Therefore $x^{*} \in T\left(x^{*}\right)$ that is $x^{*} \in F_{T}$

For the case when condition (iii) is fulfilled, the demonstration is made similarly with the proof from the second case.

Finally, we assume that the condition (iv) is verified.

Let $x^{*} \in F_{S}$ then there exists $u \in T\left(x^{*}\right)$ such that

$$
D\left(x^{*}, u, u\right) \leq \varphi\left(D\left(x^{*}, x^{*}, x^{*}\right), D\left(x^{*}, x^{*}, x^{*}\right), D\left(x^{*}, u, u\right), D\left(x^{*}, u, u\right), D\left(x^{*}, x^{*}, x^{*}\right)\right)
$$

Introducing the notation $t=D\left(x^{*}, u, u\right)$ we obtain

$$
t \leq \varphi(0,0, t, t, o)
$$

If we suppose that $t \neq 0$, then we reach the condition $t \leq \varphi(0,0, t, t, o)<t$

Thus $t=0_{\text {which means that }} u=x^{*}$. It follows that $x^{*} \in T\left(x^{*}\right)$ and so $F_{S} \subseteq F_{T}$. This completes the proof of the theorem.

\section{References:}

[1] Alina Sintamarian , Common Fixed Point Theorems For Multivalued Mappings, Seminar on Fixed Point Theory Cluj-Napoca, Volume 1, 2000, 93-102.

[2] A. Latif, I. Beg, Geometric fixed points for single and multivalued mappings, Demonstratio Math. 30 (4), 1997, 791-800

[3] B E Rhoades, A Fixed Point Theorem For Generalized Metric Spaces, Internat. J. Math. \& Math. Sci. Vol 19 No.3 (1996), $457-460$.

[4] Dhage B C, Genaralized Metric Space and Mapping with Fixed Point Bull. Cal. Math. Soc. 84, (1992), 329-336.

[5] Dhage B C, Generalized Metric Spaces and Topological Structure I, An. Stiint. Univ. A1.I. Cuza Iasi. Mat(N.S), 46, (2000), 3-24

[6] Dhage B C, On Generalized Metric Spaces and Topological Structure II, Pure. Appl. Math. Sci. 40, 1994, 37-41.

[7] Mustafa. Z and Sims. B, Some Remarks Concerning D-Metric Spaces, Proceedings of the International Conference on Fixed Point Theory and Applications, Valencia (Spain), July (2003), 189-198.

[8] C. T. Aage, J. N. Salunkhe, On Some Common Fixed Points for Contractive type Mappings in Cone Metric Spaces , Bulletin of Mathematical Analysis and Applications 1, 3(2009), 10-15.

[9] C. T. Aage, J. N. Salunkhe, Some Fixed Point Theorems in Generalized D*-Metric Spaces, Applied Sciences Vol.12, 2010, pp. 113.

[10] Seong Hoon Cho and Mi Sun Kim, Fixed Point Theorems for General Contractive Multivalued Mappings, J. Appl. Math. \& Informatics Vol. 27(2009), No. 1-2, pp. 343-350. 